\title{
Application of NETFD to the system of a localized-electron and phonons*
}

\author{
N.Arimitsu ${ }^{1}$, T.Arimitsu ${ }^{2}$ \\ 1 Department of Computer Engineering, Yokohama National University, \\ Tokiwadai, Yokohama 240-8501, Japan \\ 2 Institute of Physics, University of Tsukuba, Ibaraki 305-8571, Japan
}

Received October 6, 1999

The optical absorption spectrum in an analytically solvable model of a localized-electron and phonons is investigated using the operator algebra in NonEquilibrium Thermo Field Dynamics (NETFD). The position and the curvature of the adiabatic potentials for the ground and for the excited electronic states are assumed to differ from each other. The structures of the absorption spectra are investigated for various situations including the cases of slow modulation and of fast one. The method of NETFD is explained in order to be self-contained in solving the problem.

Key words: nonequilibrium thermo field dynamics, localized-electron, adiabatic potential, absorption spectrum, fast and slow modulations

PACS: 05.30.-d, 42.50.Ct

\section{Introduction}

In this paper, we will apply the method of NonEquilibrium Thermo Field Dynamics (NETFD) [1-5], a canonical formalism of quantum systems in far-from-equilibrium state, to an analytically soluble model of a localized-electron and phonons $[6,7]$. We will investigate the optical absorption caused by the localized-electron under the influence of phonons. In the course of the application, we will show how the operator algebra of NETFD works as if it were of quantum mechanics although the system is dissipative $[8,9]$.

The dynamics in the system of a localized-electron and phonons is specified by the Hamiltonian $[6,7]$

$$
\left.H_{\mathrm{el}-\mathrm{ph}}=\mid g\right) H_{\mathrm{g}}(g|+| u) H_{\mathrm{u}}\left(u \mid+H_{\mathrm{ph}},\right.
$$

with $(\hbar=1)$

$$
H_{\mathrm{g}}=\omega_{\mathrm{g}} b^{\dagger} b
$$

${ }^{*}$ This is an invited paper to the special issue on the problems of thermofield dynamics. 


$$
\begin{aligned}
H_{\mathrm{u}} & =\omega_{\mathrm{el}}+\omega_{\mathrm{u}} b^{\dagger} b+g_{1}\left(b^{\dagger}+b\right), \\
H_{\mathrm{ph}} & =\omega_{\mathrm{ph}} b^{\dagger} b,
\end{aligned}
$$

where $\omega_{\text {el }}$ is the energy spacing between the upper and the ground electronic states. It is assumed that the curvatures $\omega_{\mathrm{g}}$ of the adiabatic potential $H_{\mathrm{g}}$ belonging to the electronic ground state and $\omega_{\mathrm{u}}$ of $H_{\mathrm{u}}$ attached to the excited electronic level are mutually different, and that the positions of the centres of these adiabatic potentials are shifted. $H_{\mathrm{ph}}$ is the Hamiltonian for the interaction mode $\omega_{\mathrm{ph}}$ of the phonon system. $b\left(b^{\dagger}\right)$ represents the annihilation (creation) operator of the interaction mode which satisfies the canonical commutation relation

$$
\left[b, b^{\dagger}\right]=1 .
$$

The states $\mid g$ ) and $|u|$ are, respectively, the ground and the upper electronic levels which satisfy the completeness condition for the model of two electronic states, i.e.,

$$
\mid g)(g|+| u)(u \mid=1
$$

In section 2, minimal concepts and techniques of NETFD will be explained by making use of the model of a damped oscillator in order to be self-contained. An emphasis is put on the operator algebraic method in solving the problem. In comparison to the algebraic method, in appendix A, the same model will be treated by the method of path integral within NETFD. Furthermore, the model will be treated, in appendix B, with the mapped equation (the Fokker-Planck equation) by making use of the Boson coherent states within the anti-normal ordering. Before the invention of NETFD, the latter method had been mainly used in solving problems in dissipative systems (for example, see [10-12]). In section 3, an analytically soluble model of a localized-electron and phonons system will be treated by the operator algebra within NETFD in order to derive the absorption spectrum. Some discussions will be given in section 4 .

\section{Method of NETFD}

We will introduce the operator method of NETFD using the simplest but nontrivial model of a damped oscillator by comparing it with other methods in nonequilibrium statistical mechanics in terms of NETFD. For more information regarding the general method of NETFD refer to [5].

\subsection{Schrödinger equation for damped oscillator}

The dissipative dynamics of a damped oscillator is described by the Schrödinger equation

$$
\frac{\partial}{\partial t}|0(t)\rangle=-\mathrm{i} \hat{H}|0(t)\rangle
$$

for the thermal ket-vacuum $|0(t)\rangle$ within NETFD with the hat-Hamiltonian

$$
\hat{H}=H-\tilde{H}+\mathrm{i} \hat{\Pi} .
$$


Here,

$$
H=\omega a^{\dagger} a,
$$

represents the Hamiltonian of a harmonic oscillator, and

$$
\hat{\Pi}=-\kappa\left[(1+2 \bar{n})\left(a^{\dagger} a+\tilde{a}^{\dagger} \tilde{a}\right)-2(1+\bar{n}) a \tilde{a}-2 \bar{n} a^{\dagger} \tilde{a}^{\dagger}\right]-2 \kappa \bar{n},
$$

is the infinitesimal time-evolution operator describing dissipative dynamics, where $\omega$ and $\kappa$ are, respectively, the angular frequency of the oscillator and its relaxation rate. The latter is assumed to be positive. The quantity $\bar{n}$ represents the Planck distribution function defined by

$$
\bar{n}=\left(\mathrm{e}^{\omega / T}-1\right)^{-1},
$$

with temperature $T$. The operators $a, a^{\dagger}, \tilde{a}$ and $\tilde{a}^{\dagger}$ satisfy the canonical commutation relation:

$$
\left[a, a^{\dagger}\right]=1, \quad\left[\tilde{a}, \tilde{a}^{\dagger}\right]=1, \quad[a, \tilde{a}]=0, \quad \text { etc. }
$$

In NETFD, any operator $\mathcal{O}$ is accompanied by its partner (tilde) operator $\tilde{\mathcal{O}}$ where the tilde conjugation $\sim$ is defined by [13]

$$
\begin{aligned}
\left\{\mathcal{O}_{1} \mathcal{O}_{2}\right\}^{\sim} & =\tilde{\mathcal{O}}_{1} \tilde{\mathcal{O}}_{2}, \\
\left\{c_{1} \mathcal{O}_{1}+c_{2} \mathcal{O}_{2}\right\}^{\sim} & =c_{1}^{*} \tilde{\mathcal{O}}_{1}+c_{2}^{*} \tilde{\mathcal{O}}_{2}, \\
\{\tilde{\mathcal{O}}\}^{\sim} & =\mathcal{O}, \\
\left\{\mathcal{O}^{\dagger}\right\}^{\sim} & =\tilde{\mathcal{O}}^{\dagger},
\end{aligned}
$$

for operators $\mathcal{O}$ 's and complex c-numbers c's. The tilde and non-tilde operators are mutually commutative at equal time (see (12)):

$$
\left[\mathcal{O}_{1}, \tilde{\mathcal{O}}_{2}\right]=0 \text {. }
$$

The vectors $\langle 1| \mathcal{O}^{\dagger}$ and $\langle 1| \tilde{\mathcal{O}}$ in the thermal space are identical:

$$
\langle 1| \mathcal{O}^{\dagger}=\langle 1| \tilde{\mathcal{O}}
$$

where $\langle 1|$ represents the thermal bra-vacuum. We assume that the initial ket-vacuum $|0\rangle=|0(t=0)\rangle$ is specified by

$$
a|0\rangle=f \tilde{a}^{\dagger}|0\rangle,
$$

with a real quantity $f$.

The vacuum expectation value of the number operator $a^{\dagger} a$ :

$$
n(t)=\left\langle 1\left|a^{\dagger} a\right| 0(t)\right\rangle,
$$

is the one-particle distribution function satisfying the Boltzmann equation

$$
\frac{\mathrm{d}}{\mathrm{d} t} n(t)=-2 \kappa[n(t)-\bar{n}]
$$


The initial condition of the one-particle distribution function is given by

$$
n(t=0)=n=\frac{f}{1-f}
$$

which is derived by making use of the relation [5]

$$
\langle 1|a \tilde{a}| 0\rangle=\langle 1|\tilde{a} a| 0\rangle
$$

with the help of (19), (12) and (18). Note that the thermal vacuums $\langle 1|$ and $|0\rangle$ are normalized as $\langle 1 \mid 0\rangle=1$. Note also that (23) is consistent with the fact that the thermal vacuums $\langle 1|$ and $|0\rangle$ are tilde invariant:

$$
\left\langle\left. 1\right|^{\sim}=\langle 1|, \quad| 0\rangle^{\sim}=\mid 0\right\rangle .
$$

\subsection{Hat-Hamiltonian}

The hat-Hamiltonian (8) is a tildian operator satisfying

$$
\{\mathrm{i} \hat{H}\}^{\sim}=\mathrm{i} \hat{H}
$$

which is not an Hermitian operator. Note that

$$
\begin{gathered}
H^{\dagger}=H, \\
\{\hat{\Pi}\}^{\sim}=\hat{\Pi}, \\
\hat{\Pi}^{\dagger} \neq \hat{\Pi} .
\end{gathered}
$$

With the help of (18), it is an easy task to check that the hat-Hamiltonian (8) has got zero eigenvalue for the thermal bra-vacuum, i.e.,

$$
\langle 1| \hat{H}=0 .
$$

This is a manifestation of the conservation of probability, i.e., $\langle 1 \mid 0(t)\rangle=1$ (independent of time), and is indicating that the bra-vacuum $\langle 1|$ does not depend on time.

Now, we introduce a set of basic vectors [13]

$$
\mid m, \tilde{n})=|m| \mid \tilde{n}),
$$

where it is assumed that $\mid m$ ) forms the ortho-normal and complete set, i.e.,

$$
\left.\left(m \mid m^{\prime}\right)=\delta_{m, m^{\prime}}, \quad \sum_{m} \mid m\right)(m \mid=1
$$

with

$$
(m|=| m)^{\dagger},
$$


and, then, $\mid \tilde{n})=(n)^{\sim}$ also forms the ortho-normal and complete set, i.e.,

$$
\left.\left(\tilde{n} \mid \tilde{n}^{\prime}\right)=\delta_{n, n^{\prime}}, \quad \sum_{n} \mid \tilde{n}\right)(\tilde{n} \mid=1
$$

We see that the ortho-normality and the completeness for $\mid m, \tilde{n})$ are given, respectively, by

$$
\left.\left(m, \tilde{n} \mid m^{\prime}, \tilde{n}^{\prime}\right)=\delta_{m, m^{\prime}} \delta_{n, n^{\prime}}, \quad \sum_{m, n} \mid m, \tilde{n}\right)(m, \tilde{n} \mid=1
$$

with

$$
\mid m, \tilde{n})^{\dagger}=(m, \tilde{n} \mid
$$

Note that the basic vectors (30) satisfies

$$
\left.\mid m, \tilde{n})^{\sim}=\mid n, \tilde{m}\right), \quad\left(m,\left.\tilde{n}\right|^{\sim}=(n, \tilde{m} \mid .\right.
$$

Let us investigate the matrix element of the hat-Hamiltonian (8), i.e.,

$$
(k, \tilde{\ell}|\hat{H}| m, \tilde{n})=(k, \tilde{\ell}|H| m, \tilde{n})-(k, \tilde{\ell}|\tilde{H}| m, \tilde{n})+(k, \tilde{\ell}|\mathrm{i} \hat{\Pi}| m, \tilde{n}) .
$$

The first two terms are evaluated as

$$
\begin{aligned}
(k, \tilde{\ell}|H| m, \tilde{n}) & =(k|H| m)(\tilde{\ell} \mid \tilde{n}) \\
& =(k|H| m) \delta_{\ell, n},
\end{aligned}
$$

and

$$
\begin{aligned}
(k, \tilde{\ell}|\tilde{H}| m, \tilde{n}) & =(k \mid m)(\tilde{\ell}|\tilde{H}| \tilde{n}) \\
& =\delta_{k, m}(\tilde{\ell}|\tilde{H}| \tilde{n}) \\
& =\delta_{k, m}\{(\ell|H| n)\}^{\sim} \\
& =\delta_{k, m}\{(\ell|H| n)\}^{*} \\
& =\delta_{k, m}\left(n\left|H^{\dagger}\right| \ell\right) \\
& =\delta_{k, m}(n|H| \ell) .
\end{aligned}
$$

These are consistent with

$$
\left\{\left(k, \tilde{\ell}\left|\hat{H}_{0}\right| m, \tilde{n}\right)\right\}^{*}=\left(m, \tilde{n}\left|\hat{H}_{0}^{\dagger}\right| k, \tilde{\ell}\right)=\left(m, \tilde{n}\left|\hat{H}_{0}\right| k, \tilde{\ell}\right)
$$

for $\hat{H}_{0}=H-\tilde{H}$. On the other hand,

$$
\left\{\left(k, \tilde{\ell}\left|\hat{H}_{0}\right| m, \tilde{n}\right)\right\}^{*}=\left\{\left(k, \tilde{\ell}\left|\hat{H}_{0}\right| m, \tilde{n}\right)\right\}^{\sim}=-\left(\tilde{\ell}, k\left|\hat{H}_{0}\right| \tilde{n}, m\right) .
$$

(40) and (41) tells us that the complex conjugate of $\left(k, \tilde{\ell}\left|\hat{H}_{0}\right| m, \tilde{n}\right)$ can be represented by two different matrix elements. Similarly, for the third term, we have

$$
\left(m, \tilde{n}\left|\hat{\Pi}^{\dagger}\right| k, \tilde{\ell}\right)=(\ell, \tilde{k}|\hat{\Pi}| n, \tilde{m})
$$




\subsection{Density matrix}

We can represent the thermal vacuums as

$$
\begin{aligned}
|0(t)\rangle & \left.=\sum_{n, m} P_{n, m}(t) \mid n, \tilde{m}\right), \\
\langle 1| & =\sum_{n}(n, \tilde{n} \mid .
\end{aligned}
$$

The normalization of $\langle 1 \mid 0(t)\rangle$ reduces to

$$
1=\langle 1 \mid 0(t)\rangle=\sum_{k} \sum_{n, m} P_{n, m}(t)(k, \tilde{k} \mid n, \tilde{m})=\sum_{k} P_{k, k}(t),
$$

where we used the ortho-normality in (34). With the tildian hat-Hamiltonian satisfying (25), we see that the thermal ket-vacuum preserves its tilde-invariance, i.e.,

$$
|0(t)\rangle^{\sim}=|0(t)\rangle
$$

which reduces to the Hermiticy of the density matrix $P_{n, m}(t)$ [5], i.e.,

$$
P_{m, n}^{*}(t)=P_{n, m}(t) .
$$

Therefore, we see that $P_{n, n}(t)$ is real, and that, with the normalization (45),

$$
0 \leqslant P_{n, n}(t) \leqslant 1
$$

\subsection{Heisenberg equation}

Introducing the time-evolution operator $\hat{V}(t)$ by

$$
\frac{\mathrm{d}}{\mathrm{d} t} \hat{V}(t)=-\mathrm{i} \hat{H} \hat{V}(t)
$$

with the initial condition $\hat{V}(0)=1$, we can define the Heisenberg operator

$$
A(t)=\hat{V}^{-1}(t) A \hat{V}(t),
$$

which satisfies the Heisenberg equation

$$
\frac{\mathrm{d}}{\mathrm{d} t} A(t)=\mathrm{i}[\hat{H}(t), A(t)]
$$

for dissipative systems. Here,

$$
\hat{H}(t)=V(t)^{-1} \hat{H} V(t)
$$

is the hat-Hamiltonian in the Heisenberg representation. Note that the time-evolution of the unstable thermal vacuum is given by

$$
|0(t)\rangle=\hat{V}(t)|0\rangle
$$


and that

$$
\hat{V}(t)^{\sim}=\hat{V}(t) .
$$

Note also that the dissipative operators $a(t)$ etc. in the Heisenberg representation preserve the equal-time canonical commutation relation

$$
\left[a(t), a^{\dagger}(t)\right]=1, \quad\left[\tilde{a}(t), \tilde{a}^{\dagger}(t)\right]=1
$$

The equation of motion for the averaged quantity $n(t)=\left\langle 1\left|a^{\dagger}(t) a(t)\right| 0\right\rangle$ is derived by means of the Heisenberg equation (51) by taking its vacuum expectation:

$$
\frac{\mathrm{d}}{\mathrm{d} t}\left\langle 1\left|a^{\dagger}(t) a(t)\right| 0\right\rangle=\mathrm{i}\left\langle 1\left|\left[\hat{H}(t), a^{\dagger}(t) a(t)\right]\right| 0\right\rangle,
$$

which reduces to the Boltzmann equation (21) as it should be.

Let us introduce the thermal doublet notation by

$$
\begin{array}{cl}
a(t)^{\mu=1}=a(t), & a(t)^{\mu=2}=\tilde{a}^{\dagger}(t), \\
\bar{a}(t)^{\mu=1}=a^{\dagger}(t), & \bar{a}(t)^{\mu=2}=-\tilde{a}(t),
\end{array}
$$

with

$$
\begin{aligned}
a(t)=\hat{V}^{-1}(t) a \hat{V}(t), & \tilde{a}^{\dagger}(t)=\hat{V}^{-1}(t) \tilde{a}^{\dagger} \hat{V}(t), \\
a^{\dagger}(t)=\hat{V}^{-1}(t) a^{\dagger} \hat{V}(t), & \tilde{a}(t)=\hat{V}^{-1}(t) \tilde{a} \hat{V}(t) .
\end{aligned}
$$

Then, the canonical commutation relation can be written as

$$
\left[a(t)^{\mu}, \bar{a}(t)^{\nu}\right]=\delta^{\mu \nu} .
$$

Making use of the thermal doublet notation, the hat-Hamiltonian (8) reduces to

$$
\begin{aligned}
& \hat{H}=\omega \bar{a}^{\mu} a^{\mu}+\mathrm{i} \hat{\Pi}+\omega, \\
& \hat{\Pi}=-\kappa \bar{a}^{\mu} A^{\mu \nu} a^{\nu}+\kappa,
\end{aligned}
$$

with

$$
A^{\mu \nu}=\left(\begin{array}{cc}
1+2 \bar{n} & -2 \bar{n} \\
2(1+\bar{n}) & -(1+2 \bar{n})
\end{array}\right)
$$

The Heisenberg equations for the semi-free particle become

$$
\begin{aligned}
\frac{\mathrm{d}}{\mathrm{d} t} a(t)^{\mu} & =\mathrm{i}\left[\hat{H}(t), a(t)^{\mu}\right] \\
& =-\mathrm{i}\left[\omega \delta^{\mu \nu}-\mathrm{i} \kappa A^{\mu \nu}\right] a(t)^{\nu}, \\
\frac{\mathrm{d}}{\mathrm{d} t} \bar{a}(t)^{\mu} & =\mathrm{i}\left[\hat{H}(t), \bar{a}(t)^{\mu}\right] \\
& =\bar{a}(t)^{\nu} \mathrm{i}\left[\omega \delta^{\nu \mu}-\mathrm{i} \kappa A^{\nu \mu}\right] .
\end{aligned}
$$




\subsection{Annihilation and creation operators}

Let us introduce the annihilation and creation operators,

$$
\begin{array}{cl}
\gamma(t)^{\mu=1}=\gamma(t), & \gamma(t)^{\mu=2}=\tilde{\gamma}^{\text {q }}(t), \\
\bar{\gamma}(t)^{\mu=1}=\gamma^{q}(t), & \bar{\gamma}(t)^{\mu=2}=-\tilde{\gamma}(t),
\end{array}
$$

by

$$
\gamma(t)^{\mu}=B(t)^{\mu \nu} a(t)^{\nu}, \quad \bar{\gamma}(t)^{\mu}=\bar{a}(t)^{\nu} B^{-1}(t)^{\nu \mu},
$$

with the time-dependent Bogoliubov transformation:

$$
B(t)^{\mu \nu}=\left(\begin{array}{cc}
1+n(t) & -n(t) \\
-1 & 1
\end{array}\right),
$$

where $n(t)$ is the one-particle distribution function satisfying the Boltzmann equation (21). The annihilation and creation operators satisfy the canonical commutation relation

$$
\left[\gamma(t)^{\mu}, \bar{\gamma}(t)^{\nu}\right]=\delta^{\mu \nu},
$$

and annihilate the bra- and ket-vacuums at the initial time:

$$
\gamma(t)|0\rangle=0, \quad\langle 1| \tilde{\gamma}^{+}(t)=0 .
$$

The equation of motion for the thermal doublet $\gamma(t)^{\mu}$ is derived as

$$
\begin{aligned}
\frac{\mathrm{d}}{\mathrm{d} t} \gamma(t)^{\mu} & =\frac{\mathrm{d} B(t)^{\mu \nu}}{\mathrm{d} t} a(t)^{\nu}+B(t)^{\mu \nu} \frac{\mathrm{d}}{\mathrm{d} t} a(t)^{\nu} \\
& =-\mathrm{i}\left[\omega \delta^{\mu \nu}-\mathrm{i} \kappa \tau_{3}^{\mu \nu}\right] \gamma(t)^{\nu},
\end{aligned}
$$

where the matrix $\tau_{3}^{\mu \nu}$ is defined by

$$
\tau_{3}^{11}=-\tau_{3}^{22}=1, \tau_{3}^{12}=\tau_{3}^{21}=0 .
$$

For the second equality in (73), we used the Boltzmann equation (21). The solution of (73) is given by

$$
\gamma(t)^{\mu}=\exp \left\{-\mathrm{i}\left(\omega \delta^{\mu \nu}-\mathrm{i} \kappa \tau_{3}^{\mu \nu}\right)\left(t-t^{\prime}\right)\right\} \gamma\left(t^{\prime}\right)^{\nu} .
$$

Introducing the annihilation and creation operators

$$
\begin{array}{cc}
\gamma^{\mu=1}=\gamma_{t}, & \gamma^{\mu=2}=\tilde{\gamma}^{\text {q }}, \\
\bar{\gamma}^{\mu=1}=\gamma^{\text {q }}, & \bar{\gamma}^{\mu=2}=-\tilde{\gamma}_{t},
\end{array}
$$

in the Schrödinger representation by the relation

$$
\gamma(t)^{\mu}=\hat{V}^{-1}(t) \gamma_{t}^{\mu} \hat{V}(t), \quad \bar{\gamma}(t)^{\mu}=\hat{V}^{-1}(t) \bar{\gamma}_{t}^{\mu} \hat{V}(t),
$$

with $\hat{V}(t)$ being specified by (49), we can rewrite the hat-Hamiltonian (8) as

$$
\hat{H}=\omega\left(\gamma^{+} \gamma_{t}-\tilde{\gamma}^{+} \tilde{\gamma}_{t}\right)-\mathrm{i} \hat{\Pi}
$$


with

$$
\hat{\Pi}=-\kappa\left(\gamma^{\text {q}} \gamma_{t}+\tilde{\gamma}^{\text {q }} \tilde{\gamma}_{t}+2[n(t)-\bar{n}] \gamma^{\text {q }} \tilde{\gamma}^{\text {q }}\right) .
$$

It is easily derived by means of the doublet notation (62).

Substituting (79) into the quantum master equation (8), we have

$$
\begin{aligned}
\frac{\partial}{\partial t}|0(t)\rangle & =-2 \kappa[n(t)-\bar{n}] \gamma^{+} \tilde{\gamma}^{\text {क }}|0(t)\rangle \\
& =\frac{\mathrm{d} n(t)}{\mathrm{d} t} \gamma^{\text {क }} \tilde{\gamma}^{\text {के }}|0(t)\rangle .
\end{aligned}
$$

It is solved to give

$$
\begin{aligned}
|0(t)\rangle & =\exp \left[\int_{0}^{t} \mathrm{~d} t^{\prime} \frac{\mathrm{d} n\left(t^{\prime}\right)}{\mathrm{d} t^{\prime}} \gamma^{\text {q}} \tilde{\gamma}^{\text {q }}\right]|0\rangle \\
& =\exp \left[[n(t)-n(0)] \gamma^{\text {q }} \tilde{\gamma}^{\text {q}}\right]|0\rangle .
\end{aligned}
$$

This expression tells us that the vacuum is the functional of the one-particle distribution function $n(t)$. The dependence of the thermal vacuum on $n(t)$ is given by

$$
\frac{\delta}{\delta n(t)}|0(t)\rangle=\gamma^{\text {q }} \tilde{\gamma}^{+}|0(t)\rangle
$$

The Schrödinger equation (7) can be rewritten as

$$
\left\{\frac{\partial}{\partial t}+\frac{\mathrm{d} n(t)}{\mathrm{d} t} \frac{\delta}{\delta n(t)}\right\}|0(t)\rangle=0 .
$$

It is easy to see from the normal product form (79) of $\hat{H}$ that it satisfies (29), since the annihilation and creation operators satisfy

$$
\gamma_{t}|0(t)\rangle=0, \quad\langle 1| \tilde{\gamma}^{\text {q }}=0 .
$$

The hat-Hamiltonian (8) can be also written in the form

$$
\hat{H}=\omega\left(d^{\dagger} d-\tilde{d}^{\dagger} \tilde{d}\right)-\mathrm{i} \kappa\left(d^{\dagger} d+\tilde{d}^{\dagger} \tilde{d}\right)
$$

where $d^{\mu=1}=d, d^{\mu=2}=\tilde{d}^{\dagger}$ and $\bar{d}^{\mu=1}=d^{\dagger}, \bar{d}^{\mu=2}=-\tilde{d}$ are defined by

$$
d^{\mu}=\bar{B}^{\mu \nu} a^{\nu}, \quad \bar{d}^{\mu}=\bar{a}^{\nu} \bar{B}^{-1 \nu \mu},
$$

with

$$
\bar{B}^{\mu \nu}=\left(\begin{array}{cc}
1+\bar{n} & -\bar{n} \\
-1 & 1
\end{array}\right)
$$

The initial ket-thermal vacuum, $|0\rangle=|0(0)\rangle$, specified by (19), can be expressed in terms of $d$ and $\tilde{d}^{\dagger}$ as

$$
d|0\rangle=(n-\bar{n}) \tilde{d}^{\dagger}|0\rangle .
$$


It is easy to see from the diagonalized form (86) of $\hat{H}$ that

$$
\begin{aligned}
d(t) & =\hat{V}^{-1}(t) d \hat{V}(t)=d \mathrm{e}^{-(\mathrm{i} \omega+\kappa) t} \\
\tilde{d}^{\dagger}(t) & =\hat{V}^{-1}(t) \tilde{d}^{\dagger} \hat{V}(t)=\tilde{d}^{\dagger} \mathrm{e}^{-(\mathrm{i} \omega-\kappa) t} .
\end{aligned}
$$

The difference between the operators which diagonalizes $\hat{H}$ and the ones which make $\hat{H}$ in the form of normal product is one of the features of NETFD, and shows the point that the formalism is quite different from usual quantum mechanics and quantum field theory. This is a manifestation of the fact that the hat-Hamiltonian is a time-evolution generator for irreversible processes. In thermal equilibrium state, i.e., $n(t)=\bar{n}$, they coincide.

\subsection{Solution in the coherent state representation}

Applying the coherent state $(\alpha, \tilde{\beta} \mid$ defined by (190) to (83), we have

$$
\frac{\delta}{\delta n(t)}\left(\alpha, \tilde{\beta}|0(t)\rangle=\left(\alpha, \tilde{\beta}\left|\gamma^{q} \tilde{\gamma}^{\text {q }}\right| 0(t)\right\rangle\right. \text {. }
$$

With the help of

$$
\gamma^{+}=a^{\dagger}-\tilde{a}
$$

we get

$$
\begin{aligned}
\left(\alpha, \tilde{\beta}\left|\gamma^{q} \tilde{\gamma}^{\text {q }}\right| 0(t)\right\rangle= & \left(\alpha^{*} \beta-1\right)\left(\alpha, \tilde{\beta}|0(t)\rangle-\alpha^{*}(\alpha, \tilde{\beta}|a| 0(t)\rangle\right. \\
& -\beta(\alpha, \tilde{\beta}|\tilde{a}| 0(t)\rangle+(\alpha, \tilde{\beta}|\tilde{a} a| 0(t)\rangle .
\end{aligned}
$$

By making use of

$$
a=\gamma_{t}+n(t) \tilde{\gamma}^{+}
$$

we see that

$$
\begin{aligned}
(\alpha, \tilde{\beta}|a| 0(t)\rangle & =\left(\alpha, \tilde{\beta}\left|\left(\gamma_{t}+n(t) \tilde{\gamma}^{\text {q }}\right)\right| 0(t)\right\rangle \\
& =n(t)\left(\alpha, \tilde{\beta}\left|\left(\tilde{a}^{\dagger}-a\right)\right| 0(t)\right\rangle \\
& =n(t) \beta(\alpha, \tilde{\beta}|0(t)\rangle-n(t)(\alpha, \tilde{\beta}|a| 0(t)\rangle,
\end{aligned}
$$

which reduces to

$$
(\alpha, \tilde{\beta}|a| 0(t)\rangle=\frac{n(t)}{1+n(t)} \beta(\alpha, \tilde{\beta}|0(t)\rangle .
$$

Similarly, we have

$$
(\alpha, \tilde{\beta}|\tilde{a}| 0(t)\rangle=\frac{n(t)}{1+n(t)} \alpha^{*}(\alpha, \tilde{\beta}|0(t)\rangle,
$$

and

$$
(\alpha, \tilde{\beta}|\tilde{a} a| 0(t)\rangle=n(t)\left(\alpha, \tilde{\beta}|0(t)\rangle+n^{2}(t)\left(\alpha, \tilde{\beta}\left|\gamma^{\text {q }} \tilde{\gamma}^{\text {q }}\right| 0(t)\right\rangle .\right.
$$

Then, we finally obtain

$$
\left(\alpha, \tilde{\beta}\left|\gamma^{\text {के }} \tilde{\gamma}^{\text {}}\right| 0(t)\right\rangle=\left(\frac{\alpha^{*} \beta}{(1+n(t))^{2}}-\frac{1}{1+n(t)}\right)(\alpha, \tilde{\beta}|0(t)\rangle .
$$


Substituting (100), (92) becomes

$$
\frac{\delta}{\delta n(t)}\left(\alpha, \tilde{\beta}|0(t)\rangle=\left(\frac{\alpha^{*} \beta}{(1+n(t))^{2}}-\frac{1}{1+n(t)}\right)(\alpha, \tilde{\beta}|0(t)\rangle,\right.
$$

which is solved to give

$$
\begin{aligned}
(\alpha, \tilde{\beta}|0(t)\rangle & =\exp \left[\int_{n(0)}^{n(t)} \mathrm{d} n\left(\frac{\alpha^{*} \beta}{(1+n(t))^{2}}-\frac{1}{1+n(t)}\right)\right](\alpha, \tilde{\beta}|0\rangle \\
& =\frac{1+n(0)}{1+n(t)} \mathrm{e}^{\frac{n(t)-n(0)}{(1+n(0))(1+n(t))} \alpha^{*} \beta}(\alpha, \tilde{\beta}|0\rangle .
\end{aligned}
$$

Substituting the initial condition

$$
\left(\alpha, \tilde{\beta}|0\rangle=\frac{1}{1+n(0)} \mathrm{e}^{-\frac{1}{2}|\alpha|^{2}-\frac{1}{2}|\beta|^{2}+\frac{n(0)}{1+n(0)} \alpha^{*} \beta}\right.
$$

corresponding to (19) into (102), we obtain

$$
\left(\alpha, \tilde{\beta}|0(t)\rangle=\frac{1}{1+n(t)} \mathrm{e}^{-\frac{1}{2}|\alpha|^{2}-\frac{1}{2}|\beta|^{2}+\frac{n(t)}{1+n(t)} \alpha^{*} \beta} .\right.
$$

In order to see the superiority of the present operator formalism in solving the Schrödinger equation (7), we will treat it by making use of the path integral method in appendix A, and of the Fokker-Planck equation mapped in anti-normal ordering of the boson coherent state representation in appendix B.

\subsection{Initial state}

Before closing this section, let us derive the initial ket-vacuum $(\alpha, \tilde{\beta}|0\rangle$ corresponding to the statistical operator of the canonical ensemble with temperature $T_{0}$, i.e.,

$$
\left.|0\rangle=\sum_{m=0}^{\infty} P_{m, m} \mid m, \tilde{m}\right)
$$

with

$$
P_{m, m}=Z^{-1} \mathrm{e}^{-\omega m / T_{0}},
$$

where $Z$ is the partition function given by

$$
Z=1+n(0)
$$

with

$$
n(0)=\left(\mathrm{e}^{\omega / T_{0}}-1\right)^{-1}
$$

We chose for the states $\mid m, \tilde{n})$ here the number states defined by

$$
\left.\mid n, \tilde{m})=\frac{\left(a^{\dagger}\right)^{n}}{\sqrt{n !}} \frac{\left(\tilde{a}^{\dagger}\right)^{m}}{\sqrt{m !}} \mid 0, \tilde{0}\right)
$$


which satisfies the eigenfunction of the number operators $a^{\dagger} a$ and $\tilde{a}^{\dagger} \tilde{a}$ satisfying

$$
\left.\left.\left.\left.a^{\dagger} a \mid n, \tilde{m}\right)=n \mid n, \tilde{m}\right), \quad \tilde{a}^{\dagger} \tilde{a} \mid n, \tilde{m}\right)=m \mid n, \tilde{m}\right) .
$$

Note that the vacuum state $\mid 0, \tilde{0})$ is defined by

$$
a \mid 0, \tilde{0})=0, \quad \tilde{a} \mid 0, \tilde{0})=0 .
$$

With the number states, the coherent states can be represented as

$$
\left.\mid \alpha, \tilde{\beta})=\mathrm{e}^{-\frac{1}{2}|\alpha|^{2}-\frac{1}{2}|\beta|^{2}} \sum_{m=0}^{\infty} \sum_{n=0}^{\infty} \frac{\alpha^{m}}{\sqrt{m !}} \frac{\beta^{n}}{\sqrt{n !}} \mid m, \tilde{n}\right),
$$

We can evaluate $(\alpha, \tilde{\beta}|0\rangle$ as

$$
\begin{aligned}
(\alpha, \tilde{\beta}|0\rangle & =\sum_{n} P_{n, n}(\alpha, \tilde{\beta} \mid n, \tilde{n}) \\
& =(\alpha, \tilde{\beta} \mid 0, \tilde{0}) \sum_{n} P_{n, n}\left(\alpha^{*} \beta\right)^{n} \\
& =Z^{-1} \mathrm{e}^{-\frac{1}{2}|\alpha|^{2}-\frac{1}{2}|\beta|^{2}} \exp \left(\alpha^{*} \beta \mathrm{e}^{-\omega / T_{0}}\right),
\end{aligned}
$$

to give (103).

\section{Localized-electron and phonon system}

\subsection{Formulation}

The time-evolution of the system is described in NETFD by the quantum master equation (the Schrödinger equation)

$$
\frac{\partial}{\partial t}|0(t)\rangle=-\mathrm{i} \hat{H}|0(t)\rangle
$$

with the hat-Hamiltonian

$$
\hat{H}=H-\tilde{H}+\mathrm{i} \hat{\Pi},
$$

where

$$
\begin{aligned}
H & =H_{\mathrm{el}-\mathrm{ph}}-H_{\mathrm{g}} \\
& =\omega_{\mathrm{el}} N+\omega_{\mathrm{ph}} b^{\dagger} b+g_{1}\left(b^{\dagger}+b\right) N+g_{2} b^{\dagger} b N, \\
\hat{\Pi} & =-\kappa\left[(1+2 \bar{n})\left(b^{\dagger} b+\tilde{b}^{\dagger} \tilde{b}\right)-2(1+\bar{n}) \tilde{b} b-2 \bar{n} \tilde{b}^{\dagger} b^{\dagger}\right]-2 \kappa \bar{n},
\end{aligned}
$$

with

$$
g_{2}=\omega_{\mathrm{u}}-\omega_{\mathrm{g}}
$$

The operator

$$
N=c^{\dagger} c
$$


is the number operator of the localized-electron in the upper level. In deriving the expression (116) from (1), we used the correspondence

$$
\mid g)\left(g\left|\Longleftrightarrow c_{\mathrm{g}}^{\dagger} c_{\mathrm{g}}, \quad\right| u\right)\left(u \mid \Longleftrightarrow c^{\dagger} c,\right.
$$

where the annihilation and creation operators of the ground and the upper electronic states satisfy, respectively, the equal-time commutation relations

$$
\left[c_{\mathrm{g}}, c_{\mathrm{g}}^{\dagger}\right]_{+}=1, \quad\left[c, c^{\dagger}\right]_{+}=1
$$

Here, we used the anti-commutator bracket: $[X, Y]_{+}=X Y+Y X$. The completeness condition (6) reads

$$
c_{\mathrm{g}}^{\dagger} c_{\mathrm{g}}+c^{\dagger} c=1
$$

The interaction mode dissipates its energy to the rest of the phonon modes. This dissipative time-evolution is described by the generator $\hat{\Pi}$, where $\kappa$ is a positive quantity and $\bar{n}$ is given by

$$
\bar{n}=\left(\mathrm{e}^{\omega_{\mathrm{ph}} / T}-1\right)^{-1},
$$

with $T$ being the temperature of the rest of the phonon modes.

Introducing the thermal doublet notation:

$$
b^{\mu}=\left(\begin{array}{c}
b \\
\tilde{b}^{\dagger}
\end{array}\right), \quad \bar{b}^{\mu}=\left(\begin{array}{cc}
b^{\dagger}, & -\tilde{b}
\end{array}\right)
$$

we can rewrite the hat-Hamiltonian (116) in the form

$$
\hat{H}=\omega_{\mathrm{el}} \hat{N}+\bar{b}^{\mu} R^{\mu \nu} b^{\nu}+g_{1}\left(\bar{N}^{\mu} b^{\mu}+\bar{b}^{\mu} N^{\mu}\right)+\omega_{\mathrm{ph}}+g_{2} \tilde{N}+\mathrm{i} \kappa,
$$

with

$$
\begin{gathered}
\hat{N}=N-\tilde{N} \\
R^{\mu \nu}=\left(\begin{array}{cc}
\omega_{\mathrm{ph}}+g_{2} N-\mathrm{i} \kappa(1+2 \bar{n}) & 2 \mathrm{i} \kappa \bar{n} \\
-2 \mathrm{i} \kappa(1+\bar{n}) & \omega_{\mathrm{ph}}+g_{2} \tilde{N}+\mathrm{i} \kappa(1+2 \bar{n})
\end{array}\right) .
\end{gathered}
$$

The hat-Hamiltonian (125) reads

$$
\hat{H}=\omega_{\mathrm{el}} \hat{N}+\left(\bar{b}^{\mu}+\bar{\beta}^{\mu}\right) R^{\mu \nu}\left(b^{\nu}+\beta^{\nu}\right)-\bar{\beta}^{\mu} R^{\mu \nu} \beta^{\nu}+\omega_{\mathrm{ph}}+g_{2} \tilde{N}+\mathrm{i} \kappa,
$$

with

$$
\beta^{\mu}=g_{1}\left(R^{-1}\right)^{\mu \nu} N^{\nu}, \quad \bar{\beta}^{\mu}=g_{1} \bar{N}^{\nu}\left(R^{-1}\right)^{\nu \mu} .
$$

Here, we introduced the thermal doublets

$$
N^{\mu}=\left(\begin{array}{c}
N \\
\tilde{N}^{\dagger}
\end{array}\right), \quad \bar{N}^{\mu}=\left(\begin{array}{c}
\left.N^{\dagger},-\tilde{N}\right)
\end{array}\right.
$$

Inspecting that the unitary operator

$$
\hat{U}=\mathrm{e}^{-\bar{\beta}^{\mu} b^{\mu}+\bar{b}^{\mu} \beta^{\mu}},
$$


performs the shift:

$$
\hat{U} b^{\mu} \hat{U}^{-1}=b^{\mu}-\beta^{\mu}, \quad \hat{U} \bar{b}^{\mu} \hat{U}^{-1}=\bar{b}^{\mu}-\bar{\beta}^{\mu},
$$

we can transform the hat-Hamiltonian (128) into

$$
\hat{U} \hat{H} \hat{U}^{-1}=\hat{\mathcal{H}}-\bar{\beta}^{\mu} R^{\mu \nu} \beta^{\nu},
$$

where

$$
\hat{\mathcal{H}}=\hat{\mathcal{H}}_{0}+\mathrm{i} \hat{\Pi}^{\prime}
$$

with

$$
\begin{aligned}
\hat{\mathcal{H}}_{0} & =\omega_{\mathrm{el}} \hat{N}+\hat{\omega}_{\mathrm{ph}}\left(b^{\dagger} b-\tilde{b}^{\dagger} \tilde{b}\right), \\
\hat{\Pi}^{\prime} & =-\frac{\mathrm{i}}{2} g_{2}\left(b^{\dagger} b+\tilde{b}^{\dagger} \tilde{b}\right) \hat{N}+\hat{\Pi} .
\end{aligned}
$$

Here, we introduced

$$
\hat{\omega}_{\mathrm{ph}}=\omega_{\mathrm{ph}}+\frac{1}{2} g_{2}(N+\tilde{N}) .
$$

Note that $\hat{\Pi}^{\prime}$ is tilde invariant and that it can be rearranged as

$$
\hat{\Pi}^{\prime}=\bar{b}^{\mu} P^{\mu \nu} b^{\nu}+\kappa\left(1+\frac{\mathrm{i} g_{2}}{2 \kappa} \hat{N}\right)
$$

with the help of

$$
P^{\mu \nu}=\left(\begin{array}{cc}
-\frac{\mathrm{i}}{2} g_{2} \hat{N}-\kappa(1+2 \bar{n}) & 2 \kappa \bar{n} \\
-2 \kappa(1+\bar{n}) & \frac{\mathrm{i}}{2} g_{2} \hat{N}+\kappa(1+2 \bar{n})
\end{array}\right) .
$$

Corresponding to the transformation (133), we need to transform the thermal vacuum $|0(t)\rangle$ as

$$
|0(t)\rangle\rangle=\hat{U}|0(t)\rangle
$$

Then, the quantum master equation (114) becomes

$$
\left.\left.\frac{\partial}{\partial t}|0(t)\rangle\right\rangle=-\mathrm{i}\left(\hat{\mathcal{H}}-\bar{\beta}^{\mu} R^{\mu \nu} \beta^{\nu}\right)|0(t)\rangle\right\rangle
$$

Introducing the thermal vacuum $\left.\left|0^{\prime}(t)\right\rangle\right\rangle$ in the interaction representation by

$$
\left.\left.\left|0^{\prime}(t)\right\rangle\right\rangle=\mathrm{e}^{\mathrm{i} \hat{\mathcal{H}}_{0} t-\mathrm{i} \bar{\beta}^{\mu} R^{\mu \nu} \beta^{\nu} t}|0(t)\rangle\right\rangle
$$

(141) reduces to

$$
\left.\left.\frac{\partial}{\partial t}\left|0^{\prime}(t)\right\rangle\right\rangle=\hat{\Pi}^{\prime}\left|0^{\prime}(t)\right\rangle\right\rangle .
$$

Here, we used the commutativity

$$
\left[\hat{\mathcal{H}}_{0}, \hat{\Pi}^{\prime}\right]=0 .
$$


The generator $\hat{\Pi}^{\prime}$ can be diagonalized as

$$
\begin{aligned}
\hat{\Pi}^{\prime} & =-\kappa p\left(d^{\dagger} d+\tilde{d} \tilde{d}^{\dagger}\right)+\kappa\left(1+\frac{\mathrm{i} g_{2}}{2 \kappa} \hat{N}\right) \\
& =\Pi^{\prime \prime}+2 \kappa \bar{n} s
\end{aligned}
$$

with

$$
\Pi^{\prime \prime}=-\kappa p\left(d^{\dagger} d+\tilde{d}^{\dagger} \tilde{d}\right),
$$

by means of the new operators

$$
d^{\mu}=\left(\begin{array}{c}
d \\
\tilde{d}^{\dagger}
\end{array}\right), \quad \bar{d}^{\mu}=\left(\begin{array}{cc}
d^{\dagger}, & -\tilde{d}
\end{array}\right),
$$

defined through

$$
d^{\mu}=\left(Q^{-1}\right)^{\mu \nu} b^{\nu}, \quad \bar{d}^{\mu}=\bar{b}^{\nu} Q^{\nu \mu},
$$

with

$$
Q(\hat{N})^{\mu \nu}=\sqrt{\frac{1+\bar{n}}{p}}\left(\begin{array}{cc}
\frac{1}{1+s} & \frac{\bar{n}}{1+\bar{n}}(1+s) \\
1 & 1
\end{array}\right) .
$$

In (145), we introduced quantities

$$
\begin{aligned}
& p=\sqrt{\left(1+\frac{\mathrm{i} g_{2}}{2 \kappa} \hat{N}\right)^{2}+4 \bar{n} \frac{\mathrm{i} g_{2}}{2 \kappa} \hat{N}}, \\
& s=\frac{1}{2 \bar{n}}\left(1+\frac{\mathrm{i} g_{2}}{2 \kappa} \hat{N}-p\right) .
\end{aligned}
$$

Let us introduce annihilation and creation operators

$$
\gamma_{t}^{\mu}=\left(\begin{array}{c}
\gamma_{t} \\
\tilde{\gamma}_{t}^{q}
\end{array}\right), \quad \bar{\gamma}_{t}^{\mu}=\left(\begin{array}{ll}
\gamma_{t}^{q}, & -\tilde{\gamma}_{t}
\end{array}\right)
$$

by

$$
\begin{aligned}
\gamma_{t}^{\mu} & =B(t)^{\mu \nu} b^{\nu}-Z(t)^{\mu \nu} \zeta(t)^{\nu} \\
\bar{\gamma}_{t}^{\mu} & =\bar{b}^{\nu} B^{-1}(t)^{\nu \mu}-\bar{\zeta}(t)^{\nu} Z^{-1}(t)^{\nu \mu}
\end{aligned}
$$

with the time-dependent Bogoliubov transformation

$$
B(t)^{\mu \nu}=Z(t)^{\mu \eta}\left(\begin{array}{cc}
1 & -f(t) \\
-1 & 1
\end{array}\right)^{\eta \nu}
$$

where

$$
Z(t)^{\mu \nu}=\left(\begin{array}{cc}
Z_{1}(t) & 0 \\
0 & Z_{2}(t)
\end{array}\right), \quad \zeta(t)^{\nu}=\left(\begin{array}{c}
f_{0}(t) \\
-f_{1}
\end{array}\right) .
$$

Here, we introduced quantities

$$
Z_{1}(t) Z_{2}(t)=[1-f(t)]^{-1}
$$




$$
\begin{gathered}
f(t)=\frac{\frac{1}{1+s} k \mathrm{e}^{-\kappa p t}+\frac{\bar{n}}{1+\bar{n}}(1+s) \mathrm{e}^{\kappa p t}}{k \mathrm{e}^{-\kappa p t}+\mathrm{e}^{\kappa p t}}, \\
f_{0}(t)=\frac{\sqrt{\frac{p}{1+\bar{n}}} k_{0}}{k \mathrm{e}^{-\kappa p t}+\mathrm{e}^{\kappa p t}}, \\
f_{1}=\beta^{1}-\beta^{2}
\end{gathered}
$$

with

$$
k=\frac{f-\frac{\bar{n}}{1+\bar{n}}(1+s)}{\frac{1}{1+s}-f}, \quad k_{0}=\frac{\sqrt{\frac{p}{1+\bar{n}}}}{\frac{1}{1+s}-f}\left(\beta^{1}-f \beta^{2}\right) .
$$

The quantity $f$ in (161) is defined through the initial thermal state condition for the interaction mode, i.e.,

$$
b\left|0_{\mathrm{ph}}\right\rangle=f \tilde{b}^{\dagger}\left|0_{\mathrm{ph}}\right\rangle, \quad\left\langle 1_{\mathrm{ph}}\right| b=\left\langle 1_{\mathrm{ph}}\right| \tilde{b}^{\dagger} .
$$

For simplicity, we are assuming that the system of the localized-electron and of the phonon interaction mode are mutually independent at the initial time $(t=0)$ :

$$
|0(0)\rangle=\left|0_{\mathrm{el}}\right\rangle\left|0_{\mathrm{ph}}\right\rangle \text {. }
$$

The annihilation and the creation operators annihilate the thermal vacuums:

$$
\left.\left.\gamma_{t}\left|0^{\prime}(t)\right\rangle\right\rangle=0, \quad \tilde{\gamma}_{t}\left|0^{\prime}(t)\right\rangle\right\rangle=0, \quad\left\langle\langle 1| \gamma_{t}^{\text {q }}=0, \quad\left\langle\langle 1| \tilde{\gamma}_{t}^{\text {q }}=0 .\right.\right.
$$

Expressing the diagonalization operators $d, \tilde{d}^{\dagger}$ in terms of the annihilation and creation operators, i.e.,

$$
\begin{gathered}
d=\sqrt{\frac{1+\bar{n}}{p}}\left[Z_{2}(t) \frac{1-\bar{n} s}{1+\bar{n}} \gamma_{t}+Z_{1}(t)\left(f(t)-\frac{\bar{n}(1+s)}{1+\bar{n}}\right) \tilde{\gamma}_{t}^{\text {q }}-Z_{1}(t) Z_{2}(t) e_{+}(t)\right], \\
\tilde{d}^{\dagger}=\sqrt{\frac{1+\bar{n}}{p}}\left[-Z_{2}(t) \frac{s}{1+s} \gamma_{t}+Z_{1}(t)\left(-f(t)+\frac{1}{1+s}\right) \tilde{\gamma}_{t}^{\text {q }}+Z_{1}(t) Z_{2}(t) e_{-}(t)\right],
\end{gathered}
$$

with

$$
\begin{aligned}
& e_{+}(t)=f_{0}(t)-f(t) f_{1}-\frac{\bar{n}}{1+\bar{n}}(1+s)\left[f_{0}(t)-f_{1}\right] \\
& e_{-}(t)=f_{0}(t)-f(t) f_{1}-\frac{1}{1+s}\left[f_{0}(t)-f_{1}\right]
\end{aligned}
$$

and substituting them in (146), we have got the time-evolution operator $\hat{\Pi}^{\prime \prime}$ in the form:

$$
\hat{\Pi}^{\prime \prime}=\kappa(1+\bar{n})\left\{2 Z_{2}(t)^{2} \frac{s(1-\bar{n} s)}{(1+s)(1+\bar{n})} \tilde{\gamma}_{t} \gamma_{t}\right.
$$




$$
\begin{aligned}
& +Z_{1}(t) Z_{2}(t)\left(\frac{s}{1+s}\left[f(t)-\frac{\bar{n}}{1+\bar{n}}(1+s)\right]\right. \\
& \left.+\left[f(t)-\frac{1}{1+s}\right] \frac{1-\bar{n} s}{1+\bar{n}}\right)\left(\tilde{\gamma}_{t}^{\text {q }} \tilde{\gamma}_{t}+\gamma_{t}^{\text {q }} \gamma_{t}\right) \\
& +2 Z_{1}(t)^{2}\left[f(t)-\frac{1}{1+s}\right]\left[f(t)-\frac{\bar{n}}{1+\bar{n}}(1+s)\right] \gamma_{t}^{\text {q }} \tilde{\gamma}_{t}^{\text {q }} \\
& +2 Z_{1}(t) Z_{2}(t) \frac{s}{1+s}\left[f(t)-\frac{\bar{n}}{1+\bar{n}}(1+s)\right] \\
& \left.+2 Z_{1}(t)^{2} Z_{2}(t)^{2}\left[e_{+}(t) \tilde{e}_{-}(t)+e_{-}(t) \tilde{e}_{+}(t)\right]\right\} .
\end{aligned}
$$

Expression (169) is the normal ordered form of the generator $\hat{\Pi}^{\prime \prime}$, whereas expression (145) with (146) is the diagonalized form of the generator. Note that, in the usual quantum mechanics or quantum field theory, the operator which diagonalizes the Hamiltonian and the one which defines the normal ordering are the same. It is one of the special features of transient nonequilibrium situations that the diagonalizing operator of hat-Hamiltonian is different from its normal ordering operator as has been mentioned in the previous section.

The thermal vacuum ket-vector of the localized-electron system for arbitrary time $t(>0)$ is given by

$$
\left|0_{\mathrm{el}}(t)\right\rangle=\left\langle 1_{\mathrm{ph}} \mid 0(t)\right\rangle=G(t, \hat{N})\left|0_{\mathrm{el}}\right\rangle,
$$

where we have introduced

$$
G(t, \hat{N})=\mathrm{e}^{\mathrm{i} \bar{\beta}^{\mu} R^{\mu \nu} \beta^{\nu} t-\mathrm{i} \omega_{\mathrm{el}} \hat{N} t+2 \kappa \bar{n} s t} S(t, \hat{N}),
$$

with

$$
S(t, \hat{N})=\left\langle\left\langle 1_{\mathrm{ph}}\left|\mathrm{e}^{\left[\left(-\mathrm{i} \hat{\mathrm{\omega}}_{\mathrm{ph}}-\kappa p\right) d^{\dagger} d+\left(\mathrm{i} \hat{\mathrm{p}}_{\mathrm{ph}}-\kappa p\right) \tilde{d}^{\dagger} \tilde{d}\right] t}\right| 0_{\mathrm{ph}}\right\rangle\right\rangle .
$$

The function $S(t, \hat{N})$ satisfies the differential equation

$$
\begin{aligned}
\frac{\mathrm{d} S(t, \hat{N})}{\mathrm{d} t}=2 \kappa(1+\bar{n}) Z_{1}(t) Z_{2}(t) \frac{s}{1+s}\left[f(t)-\frac{\bar{n}}{1+\bar{n}}(1+s)\right] S(t, \hat{N}) \\
-\frac{1+\bar{n}}{p} Z_{1}(t)^{2} Z_{2}(t)^{2}\left[\left(-\kappa p-\mathrm{i} \hat{\omega}_{\mathrm{ph}}\right) e_{+}(t) \tilde{e}_{-}(t)\right. \\
\left.\quad+\left(-\kappa p+\mathrm{i} \hat{\omega}_{\mathrm{ph}}\right) e_{-}(t) \tilde{e}_{+}(t)\right] S(t, \hat{N}), \\
=-\left[\frac{\mathrm{d}}{\mathrm{d} t} \ln \left(1-r+r \mathrm{e}^{-2 \kappa p t}\right)-E(t)\right] S(t, \hat{N}),
\end{aligned}
$$

where

$$
\begin{aligned}
r= & n s-\bar{n} s p^{-1}(1-n s) \\
E(t)=- & (1+\bar{n}) Z_{1}^{2}(t) Z_{2}^{2}(t)\left[\left(-\kappa p-\mathrm{i} \hat{\omega}_{\mathrm{ph}}\right) e_{+}(t) \tilde{e}_{-}(t)\right. \\
& \left.+\left(-\kappa p+\mathrm{i} \hat{\omega}_{\mathrm{ph}}\right) e_{-}(t) \tilde{e}_{+}(t)\right] / p .
\end{aligned}
$$


The differential equation (173) was derived by making use of the similar method given in subsection 2.6. It can be solved to give

$$
S(t, \hat{N})=\frac{1}{1-r+r \mathrm{e}^{-2 \kappa p t}} \exp \left[\int_{0}^{t} \mathrm{~d} t^{\prime} E\left(t^{\prime}\right)\right] .
$$

\subsection{Absorption spectrum}

The absorption spectrum of the system is given by the imaginary part of an intensity distribution function $\phi(\omega)$ defined by

$$
\phi(\omega)=\phi^{\prime}(\omega)+\mathrm{i} \phi^{\prime \prime}(\omega)=\int_{0}^{\infty} \mathrm{d} t \mathrm{e}^{\mathrm{i} \omega t} \Phi(t)
$$

where $\Phi(t)$ is the auto-correlation function (the response function)

$$
\Phi(t)=\mathrm{i}\left\langle 1_{\mathrm{el}}\left|\left[c(t), c^{\dagger}(0)\right]\right| 0_{\mathrm{el}}\right\rangle+\text { c.c. },
$$

with $c(t)=\mathrm{e}^{-\mathrm{i} \hat{H} t} c \mathrm{e}^{\mathrm{i} \hat{H} t}$. With the help of $G(t, \hat{N})$ given in (171), the auto-correlation function (178) can be expressed as

$$
\Phi(t)=\mathrm{i} G(t, \hat{N}=1)+\text { c.c. }
$$

with $N=1$ and $\tilde{N}=0$.

The absorption spectrum $\phi^{\prime \prime}(\omega)$ in (177) is shown in figures 1 and 2 for several values for $g_{1}$ and $g_{2}$ with $\kappa=0.001, n=\bar{n}=5$. The energies or the angular frequencies are scaled by $\omega_{\mathrm{g}}$, i.e., the curvature of the adiabatic potential belonging to the electronic ground state.

In figure 1 , fixing $g_{1}=0.1$, we displayed the absorption spectrum by changing the parameter $g_{2}$, i.e., a) 0.2 , b) 0.1 , c) 0.05 and d) 0.01 . These situations can be categorized by the parameter $\alpha$ defined by

$$
\alpha=\frac{g_{2} \sqrt{\bar{n}(\bar{n}+1)}}{2 \kappa}
$$

The cases for $\alpha>1$ are categorized as slower modulation, whereas the cases for $\alpha<1$ as faster modulation. The situations in figure 1 correspond to the cases of slower modulation, i.e., $\alpha=$ a) 547, b) 274, c) 137 and d) 27.4. There appear phonon side bands for larger values of $\alpha$ due to the dynamics of phonon interaction mode. We can recognize five phonon side bands for a), three bands for b) and one band for c). The side bands disappear for $\mathrm{d}$ ). The reason for the disappearance of side bands is that the faster the modulation becomes the dynamics of phonon is smeared out even for $\alpha>1$.

From a) to c) in figure 1, we observe that the space between adjacent phonon bands is equal to $g_{2}$. It can be interpreted by a semi-classical argument as follows. Putting $b=\xi \mathrm{e}^{-\mathrm{i} \psi}$ and neglecting dissipation, we have

$$
\begin{aligned}
& H_{\mathrm{g}}=\omega_{\mathrm{g}} \xi^{2}, \\
& H_{\mathrm{u}}=\omega_{\mathrm{el}}+\omega_{\mathrm{u}}\left(\xi+\frac{g_{1}}{\omega_{\mathrm{u}}} \cos \psi\right)^{2}-\frac{g_{1}^{2}}{\omega_{\mathrm{u}}} \cos ^{2} \psi .
\end{aligned}
$$



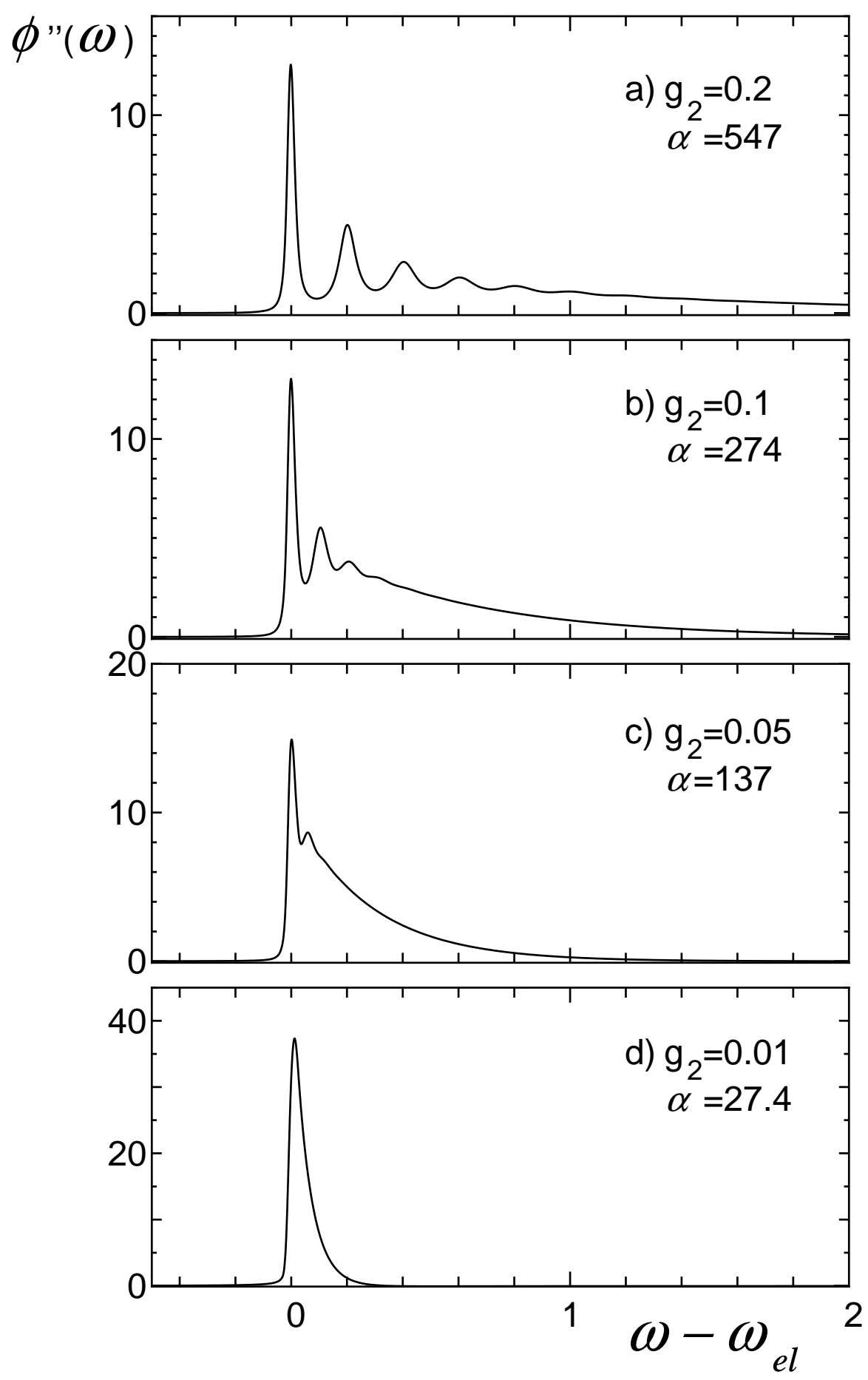

Figure 1. The absorption spectrum $\phi^{\prime \prime}(\omega)$ for $\kappa=0.001, g_{1}=0.1$ and $n=\bar{n}=5$ in the cases that $g_{2}$ is equal to a) 0.2 , b) 0.1 , c) 0.05 and d) 0.01 . These cases are specified, respectively, by the parameter $\alpha$, i.e., a) 547, b) 274, c) 137 and d) 27.4. The energies or the angular frequencies are scaled by $\omega_{\mathrm{g}}$. 


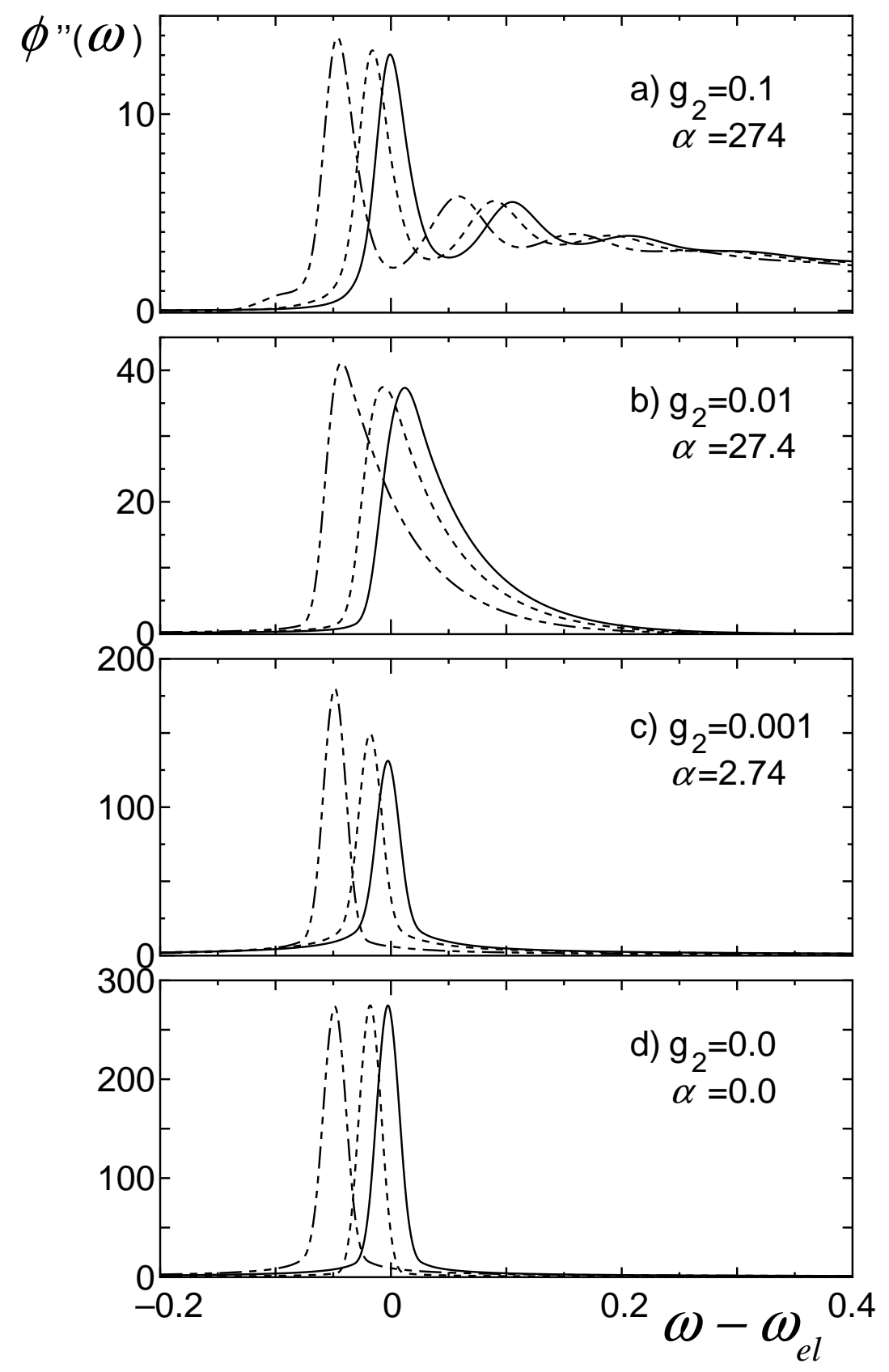

Figure 2. The absorption spectrum $\phi^{\prime \prime}(\omega)$ for $\kappa=0.001$ and $n=\bar{n}=5$, in the cases that $g_{2}$ is equal to a) 0.1, b) 0.01 , c) 0.001 and d) 0 . Correspondingly, the parameter $\alpha$ is a) 274, b) 27.4, c) 2.74, and d) 0. In each figure, the solid line represents the case $g_{1}=0.1$, the dashed line $g_{1}=0.3$, and the two-dotted dashed line $g_{1}=0.5$. The energies or the angular frequencies are scaled by $\omega_{\mathrm{g}}$. 
The absorption spectrum is, then, given by sum of delta functions corresponding to the transitions from the lower ladder with the energy space $\omega_{\mathrm{g}}$ to the upper one with the energy space $\omega_{\mathrm{u}}$. The upper ladder starts with the energy $\omega_{\mathrm{el}}-g_{1}^{2} \cos ^{2} \psi / \omega_{\mathrm{u}}$. Therefore, the positions of the absorption spectrum are mainly given by the transitions from the first step of the lower ladder to the first step of the upper ladder, from the second step of the lower ladder to the second step of the upper ladder and so on, i.e.,

$$
\omega-\omega_{\mathrm{el}}=-\frac{g_{1}^{2} \cos ^{2} \psi}{\omega_{\mathrm{g}}+g_{2}}+g_{2} \ell, \quad \ell=0,1,2, \cdots .
$$

They are accompanied by other transitions between different steps of the lower and the upper ladders. The expression (183) explains that the space between side bands are given by $g_{2}=\omega_{\mathrm{u}}-\omega_{\mathrm{g}}$.

In figure 2, the absorption spectrums are displayed by changing the value of $g_{2}$, i.e., a) 0.1, b) 0.01 , c) 0.001 , d) 0.0. Each figure contains three lines for different values of $g_{1}$, i.e., 0.1 (solid curve), 0.3 (dashed line) and 0.5 (two-dotted dashed line). Note that the solid lines in a) and b) of figure 2 are, respectively, equal to the lines in b) and d) of figure 1 .

As can be seen from (183), the shifts of peaks due to $g_{1}$ are given by $-g_{1}^{2} \cos ^{2} \psi /$ $\left(\omega_{\mathrm{g}}+g_{2}\right)$. It can be ascertained by measuring the shifts in figure 2 with $\cos ^{2} \psi \simeq 0.2$. It tells us that the measurement of the shift of peaks gives us the phase $\psi$ if we know the value of $g_{1} / \omega_{\mathrm{g}}$. For slow modulation like a) with $\alpha=274$, we observe several side bands due to the dynamics of phonon interaction mode. The spaces between the peaks of the side bands are given by $g_{2}$ as has been explained above. The change of the absorption spectrums from c) to d) can be interpreted by the concept of motional narrowing.

\section{Discussion}

We treated, by making use of the operator algebra within the formalism of NETFD, the model of a localized-electron and phonons system in terms of the adiabatic potential with a shift of position of the excited state compared with the ground state in addition to the change of its curvature. Compared to the old analysis [12] where the mapping technique by means of the boson coherent state representation was used to solve the problem (see appendix B), the technical convenience of the operator algebra in NETFD, which is very much similar to that of the usual quantum mechanics, enables us to treat open systems in far-from-equilibrium state simpler and more transparent $[8,9,14-17]$.

In addition to the technical convenience, NETFD contains quite a few conceptual advantages in constructing quantum field theory for dissipative systems by implanting the concepts developed in nonequilibrium thermodynamics. We would like to close this paper by mentioning some potentialities of NETFD for future developments.

The expression (82) tells us that the time-evolution of the unstable vacuum is realized by the condensation of $\gamma_{k}^{q} \tilde{\gamma}_{k}^{q}$-pairs into the vacuum. Here, we put the 
subscript $k$ for wave vector and/or other degrees of freedom appearing in quantum field theoretical arguments. The attractive expression (82), which was obtained first in [18], led us to the notion of a mechanism named the spontaneous creation of dissipation [19-23]. We can obtain the result (82) only by algebraic manipulations. It also shows that the vacuum is the functional of the one-particle distribution function $n_{k}(t)$. The dependence of the thermal vacuum on $n_{k}(t)$ is given by

$$
\frac{\delta}{\delta n_{k}(t)}|0(t)\rangle=\gamma_{k}^{\text {q }} \tilde{\gamma}_{k}^{\text {q }}|0(t)\rangle
$$

We see that the vacuum $|0(t)\rangle$ represents a state which consists of a macroscopic object described by the one-particle distribution function $n_{k}(t)$. The master equation (7) can be rewritten as

$$
\left\{\frac{\partial}{\partial t}+\int \mathrm{d}^{3} k \frac{\mathrm{d} n_{k}(t)}{\mathrm{d} t} \frac{\delta}{\delta n_{k}(t)}\right\}|0(t)\rangle=0
$$

This shows that the vacuum, in this case, is migrating in the super-representation space spanned by the one-particle distribution function $\left\{n_{k}(t)\right\}$ with the velocity $\left\{\mathrm{d} n_{k}(t) / \mathrm{d} t\right\}$ as a conserved quantity [24].

The framework of NETFD has been extended (for example, see [5]) to take account of the aspects of the Langevin equation and the stochastic Liouville equation. There, again NETFD allowed us to construct a unified canonical theory of quantum stochastic operators, i.e., a unified system of quantum stochastic differential equations. The stochastic Liouville equations both of the Ito and of the Stratonovich types were introduced as the stochastic Schrödinger equation in the Schrödinger representation. Whereas, the Langevin equations both of the Ito and of the Stratonovich types were constructed as the Heisenberg equation of motion with the help of the time-evolution generator of corresponding stochastic Liouville equations. The Ito formula was derived for quantum systems. The problem, why the track of an injected particle in the cloud chamber keeps its width finite, was investigated dynamically by means of the quantum stochastic calculus within the method of NETFD [25]. The measurement (a continuous non-demolition measurement) of the incident particle by ionizing the gas molecules in the cloud chamber is interpreted as a stochastic agitation due to the quantum Brownian motion [26]. It was shown that the watch-dog effect, i.e., the continuous non-demolition measurement of the injected particle by the gas molecules, prevents the wave packet spreading out in contrast with the case of a free particle.

Kinetics and hydrodynamics of highly nonequilibrium and strongly coupled quantum systems were investigated [27] by making use of NETFD based on the Zubarev's nonequilibrium statistical operator method $[28,29]$. Within this method, two different levels of describing the kinetics and hydrodynamics of dense quantum nuclear systems were considered: strongly coupled states and quark-gluon plasma. We expect that the consistent description of kinetics and hydrodynamics may provide us with a wider viewpoint for the dynamics in far-from equilibrium states. 


\section{A. Path integral method}

The kernel

$$
K\left(\alpha, \beta, t ; \alpha^{\prime}, \beta^{\prime}, t^{\prime}\right)=\left(\alpha, \tilde{\beta}\left|\mathrm{e}^{-\mathrm{i} \hat{H}\left(t-t^{\prime}\right)}\right| \alpha^{\prime}, \tilde{\beta}^{\prime}\right),
$$

defined by

$$
\left(\alpha, \tilde{\beta}|0(t)\rangle=\int \frac{\mathrm{d}^{2} \alpha^{\prime}}{\pi} \int \frac{\mathrm{d}^{2} \beta^{\prime}}{\pi} K\left(\alpha, \beta, t ; \alpha^{\prime}, \beta^{\prime}, 0\right)\left(\alpha^{\prime}, \tilde{\beta}^{\prime}|0\rangle,\right.\right.
$$

can be expressed in terms of path integral. Here, we introduced the coherent state by

$$
\left.\mid \alpha, \tilde{\beta})=\mathrm{e}^{\alpha a^{\dagger}-\alpha^{*} a} \mathrm{e}^{\beta^{*} \tilde{a}^{\dagger}-\beta \tilde{a}} \mid 0, \tilde{0}\right),
$$

with

$$
\begin{aligned}
a \mid \alpha, \tilde{\beta}) & \left.=\alpha \mid \alpha, \tilde{\beta}), \quad \tilde{a} \mid \alpha, \tilde{\beta})=\beta^{*} \mid \alpha, \tilde{\beta}\right), \\
\left(\alpha, \tilde{\beta} \mid a^{\dagger}\right. & =\left(\alpha, \tilde{\beta} \mid \alpha^{*}, \quad\left(\alpha, \tilde{\beta} \mid \tilde{a}^{\dagger}=(\alpha, \tilde{\beta} \mid \beta .\right.\right.
\end{aligned}
$$

By making use of

$$
\left(\alpha_{n}, \tilde{\beta}_{n} \mid \alpha_{n-1}, \tilde{\beta}_{n-1}\right)=\mathrm{e}^{-\frac{1}{2}\left|\alpha_{n}\right|^{2}-\frac{1}{2}\left|\alpha_{n-1}\right|^{2}+\alpha_{n}^{*} \alpha_{n-1}} \mathrm{e}^{-\frac{1}{2}\left|\beta_{n-1}\right|^{2}-\frac{1}{2}\left|\beta_{n}\right|^{2}+\beta_{n-1}^{*} \beta_{n}},
$$

and

$$
\begin{aligned}
& h_{n, n-1}\left(\alpha_{n}, \tilde{\beta}_{n} \mid \alpha_{n-1}, \tilde{\beta}_{n-1}\right)=\left(\alpha_{n}, \beta_{n}|\hat{H}| \alpha_{n-1}, \tilde{\beta}_{n-1}\right) \\
& =\left\{\omega \alpha_{n}^{*} \alpha_{n-1}-\omega \beta_{n} \beta_{n-1}^{*}-\mathrm{i} \kappa\left[(1+2 \bar{n})\left(\alpha_{n}^{*} \alpha_{n-1}+\beta_{n} \beta_{n-1}^{*}\right)\right.\right. \\
& \left.\left.\quad-2(1+\bar{n}) \alpha_{n-1} \beta_{n-1}^{*}-2 \bar{n} \alpha_{n}^{*} \beta_{n}\right]-2 \mathrm{i} \kappa \bar{n}\right\}\left(\alpha_{n}, \tilde{\beta}_{n} \mid \alpha_{n-1}, \tilde{\beta}_{n-1}\right),
\end{aligned}
$$

the path integral

$$
\begin{aligned}
& K\left(\alpha, \beta, t ; \alpha^{\prime}, \beta^{\prime}, t^{\prime}\right) \\
& =\lim _{N \rightarrow \infty}\left(\prod_{i=1}^{N-1} \int \frac{\mathrm{d}^{2} \alpha_{i}}{\pi}\right)\left(\prod_{j=1}^{N-1} \int \frac{\mathrm{d}^{2} \beta_{j}}{\pi}\right) \\
& \quad \times\left(\alpha_{N}, \tilde{\beta}_{N} \mid \alpha_{N-1}, \tilde{\beta}_{N-1}\right) \cdots\left(\alpha_{1}, \tilde{\beta}_{1} \mid \alpha_{0}, \tilde{\beta}_{0}\right) \exp \left[-\mathrm{i} \Delta t \sum_{n=1}^{N-1} h_{n, n-1}\right] \\
& =\quad \mathrm{e}^{-\frac{1}{2}|\alpha|^{2}-\frac{1}{2}\left|\alpha^{\prime}\right|^{2}-\frac{1}{2}|\beta|^{2}+\frac{1}{2}\left|\beta^{\prime}\right|^{2}} \int \mathcal{D}^{2} \alpha \int \mathcal{D}^{2} \beta \\
& \times \exp \left[\mathrm { i } \int _ { t ^ { \prime } } ^ { t } \mathrm { d } t \left[\mathrm{i} \alpha^{*}(t) \dot{\alpha}(t)-\mathrm{i} \beta^{*}(t) \dot{\beta}(t)\right.\right. \\
& \quad-\alpha(t)\left\{\omega \alpha^{*}(t)-\mathrm{i} \kappa(1+2 \bar{n}) \alpha^{*}(t)+2 \mathrm{i} \kappa(1+\bar{n}) \beta^{*}(t)\right\} \\
& \left.\left.\quad-\beta(t)\left\{-\omega \beta^{*}(t)-\mathrm{i} \kappa(1+2 \bar{n}) \beta^{*}(t)+2 \mathrm{i} \kappa \bar{n} \alpha^{*}(t)\right\}-2 \kappa \bar{n}\right]\right],
\end{aligned}
$$

can be solved with the help of the equations of motion

$$
\frac{\mathrm{d}}{\mathrm{d} t}\left(\alpha^{*}(t), \beta^{*}(t)\right)^{\mu}=\left(\alpha^{*}(t), \beta^{*}(t)\right)^{\nu} \mathrm{i}\left[\omega \delta^{\nu \mu}-\mathrm{i} \kappa A^{\nu \mu}\right],
$$


with the boundary conditions

$$
\alpha^{*}(t)=\alpha^{*}, \quad \alpha\left(t^{\prime}\right)=\alpha^{\prime}, \quad \beta^{*}\left(t^{\prime}\right)=\beta^{*}, \quad \beta(t)=\beta .
$$

The matrix $A$ is given by (64). Note that (194) is equivalent to the Heisenberg equation (66).

The result is

$$
\begin{aligned}
& K\left(\alpha, \beta, t ; \alpha^{\prime}, \beta^{\prime}, t^{\prime}\right)=\nu\left(t-t^{\prime}\right) \exp \left[-\frac{1}{2}|\alpha|^{2}-\frac{1}{2}\left|\alpha^{\prime}\right|^{2}-\frac{1}{2}|\beta|^{2}-\frac{1}{2}\left|\beta^{\prime}\right|^{2}\right. \\
& \left.+k_{i}\left(t-t^{\prime}\right) \beta^{\prime *} \alpha^{\prime}+k_{f}\left(t-t^{\prime}\right) \alpha^{*} \beta+\ell\left(t-t^{\prime}\right) \alpha^{*} \alpha^{\prime}+\ell^{*}\left(t-t^{\prime}\right) \beta^{\prime *} \beta\right],
\end{aligned}
$$

where

$$
\begin{aligned}
k_{i}(t) & =(1+\bar{n}) \nu(t)\left(1-\mathrm{e}^{-2 \kappa t}\right), \\
k_{f}(t) & =\bar{n} \nu(t)\left(1-\mathrm{e}^{-2 \kappa t}\right), \\
\ell(t) & =\nu(t) \mathrm{e}^{-\mathrm{i} \omega t-\kappa t}
\end{aligned}
$$

with

$$
\nu(t)=\left[(1+\bar{n})-\bar{n} \mathrm{e}^{-2 \kappa t}\right]^{-1} .
$$

Then, by a lengthy but straightforward calculation, we finally obtain

$$
\begin{aligned}
(\alpha, \tilde{\beta}|0(t)\rangle & =\int \frac{\mathrm{d}^{2} \alpha^{\prime}}{\pi} \int \frac{\mathrm{d}^{2} \beta^{\prime}}{\pi} K\left(\alpha, \beta, t ; \alpha^{\prime}, \beta^{\prime}, 0\right)\left(\alpha^{\prime}, \tilde{\beta}^{\prime}|0\rangle\right. \\
& =\frac{1}{1+n(t)} \mathrm{e}^{-\frac{1}{2}|\alpha|^{2}-\frac{1}{2}|\beta|^{2}+\frac{n(t)}{1+n(t)} \alpha^{*} \beta}
\end{aligned}
$$

which is just the same as (104) as it should be. Here, we used (103) for the initial condition whose derivation is given in section 2.7 .

\section{B. Mapped equation by anti-normal ordering}

Introducing the boson coherent state representation of the anti-normal ordering [30-32] through

$$
\left.|0(t)\rangle=\int \frac{\mathrm{d}^{2} z}{\pi} f(z, z, t) \mid z, \tilde{z}\right),
$$

with the boson coherent state $\mid z, \tilde{z})$, defined by (189), we can map the Schrödinger equation (7) into a partial differential equation for the c-number function $f(z, t)=$ $f(z, z, t)$ as $[33]$

$$
\frac{\partial}{\partial t} f(z, t)=\left[-\mathrm{i} \omega\left(\frac{\partial}{\partial z^{*}} z^{*}-\text { c.c. }\right)+\kappa\left(\frac{\partial}{\partial z^{*}} z^{*}+\text { c.c. }\right)+2 \kappa \bar{n} \frac{\partial}{\partial z^{*}} \frac{\partial}{\partial z}\right] f(z, t) .
$$

Now let us investigate the Fokker-Planck equation (203). With the transformation

$$
F(z, t)=\mathrm{e}^{\mathrm{i} t \omega\left(\frac{\partial}{\partial z^{*}} z^{*}-\frac{\partial}{\partial z} z\right)} f(z, t)
$$


the Fokker-Planck equation (203) is transformed into

$$
\frac{\partial}{\partial t} F(\xi, t)=2 \kappa\left(\frac{\partial}{\partial \xi} \xi+\bar{n} \frac{\partial}{\partial \xi} \xi \frac{\partial}{\partial \xi}\right) F(\xi, t),
$$

where $\xi=|z|^{2}$. Putting

$$
F(\xi, t)=R(\xi / \bar{n}) \mathrm{e}^{-2 \kappa \lambda t},
$$

in (205) and changing the variable as $\zeta=\xi / \bar{n}$, we have an eigenvalue equation for the right-hand side eigenfunctions

$$
\zeta R^{\prime \prime}(\zeta)+(1+\zeta) R^{\prime}(\zeta)+R(\zeta)=-\lambda R(\zeta)
$$

The differential equation (an eigenvalue equation for the left-hand side eigenfunctions) adjoint of (207) turns out to be

$$
\zeta L^{\prime \prime}(\zeta)+(1-\zeta) L^{\prime}(\zeta)=-\lambda L(\zeta)
$$

Note that $R(\zeta)$ is related to $L(\zeta)$ by

$$
R(\zeta)=\mathrm{e}^{-\zeta} L(\zeta)
$$

Now, we remember that the Laguerre polynomials defined by

$$
\sum_{\ell=0}^{\infty} L_{\ell}(\zeta) x^{\ell}=\frac{1}{1-x} \mathrm{e}^{-\zeta \frac{x}{1-x}}
$$

or

$$
L_{\ell}(\zeta)=\frac{1}{\ell !} \mathrm{e}^{\zeta} \frac{\mathrm{d}^{\ell}}{\mathrm{d} \zeta^{\ell}}\left(\mathrm{e}^{-\zeta} \zeta^{\ell}\right)=\sum_{k=0}^{\ell}(-1)^{k}\left(\begin{array}{l}
\ell \\
k
\end{array}\right) \frac{\zeta^{k}}{k !},
$$

satisfy the differential equation

$$
\zeta L_{\ell}^{\prime \prime}(\zeta)+(1-\zeta) L_{\ell}^{\prime}(\zeta)+\ell L_{\ell}(\zeta)=0 .
$$

For example, $L_{n}(\zeta)^{\prime}$ 's are given by

$$
L_{0}(\zeta)=1, \quad L_{1}(\zeta)=1-\zeta, \quad L_{2}(\zeta)=1-2 \zeta+\zeta^{2} / 2 .
$$

We notice in comparison of (212) with (207) and (208) that the eigenvalue $\lambda$ should be

$$
\lambda=\ell, \quad(\ell=0,1,2, \ldots),
$$

and that the right and the left eigenfunctions belonging to the same eigenvalue, say $\ell$, are given respectively by

$$
R_{\ell}(\zeta)=L_{\ell}(\zeta) \mathrm{e}^{-\zeta}, \quad L_{\ell}(\zeta)=L_{\ell}(\zeta)
$$


These eigenfunctions form an ortho-normal complete set satisfying

$$
\begin{gathered}
\int_{0}^{\infty} \mathrm{d} \zeta L_{\ell}(\zeta) R_{\ell^{\prime}}(\zeta)=\delta_{\ell, \ell^{\prime}}, \\
\sum_{\ell=0}^{\infty} R_{\ell}(\zeta) L_{\ell}\left(\zeta^{\prime}\right)=\delta\left(\zeta-\zeta^{\prime}\right) .
\end{gathered}
$$

Note that the right-hand side eigenfunctions $R_{\ell}(\zeta)$ are of $L_{2}\left(\mathbf{R}_{+}\right)$, whereas the lefthand side eigenfunctions $L_{\ell}(\zeta)$ are not. We may say that $R_{\ell}(\zeta)$ and $L_{\ell}(\zeta)$ belong respectively to the nuclear space and its conjugate space in the Gel'fand triplet (or the rigged Hilbert space). Note that the left eigenfunction $L_{0}(\zeta)=1$ corresponds to the thermal bra-vacuum $\langle 1|$. This is the reason why we put one for the index of the bra-vacuum instead of zero.

Let us solve (205) by expanding the desired function $F(\xi, t)$ as

$$
F(\zeta \bar{n}, t)=\sum_{\ell=0}^{\infty} a_{\ell} R_{\ell}(\zeta) \mathrm{e}^{-2 \kappa \ell t}
$$

with the initial condition

$$
F(\xi, 0)=f(\xi, 0)=\frac{1}{n(0)} \mathrm{e}^{-\xi / n(0)}
$$

which is derived as follows. Substituting (103) into

$$
\begin{aligned}
(\alpha \alpha|0\rangle & =\int \frac{\mathrm{d}^{2} z}{\pi} f(z, z)(\alpha, \alpha \mid z, z) \\
& =\int \frac{\mathrm{d}^{2} z}{\pi} f(z, z) \mathrm{e}^{-(z-\alpha)\left(z^{*}-\alpha^{*}\right)},
\end{aligned}
$$

we have the integral equation

$$
\frac{1}{1+n(0)} \mathrm{e}^{-x^{2} /(1+n(0))} \mathrm{e}^{-y^{2} /(1+n(0))}=\int \frac{\mathrm{d} x_{1} \mathrm{~d} y_{1}}{\pi} f\left(x_{1}, y_{1}\right) \mathrm{e}^{-\left(x_{1}-x\right)^{2}} \mathrm{e}^{-\left(y_{1}-y\right)^{2}},
$$

where we have changed the integration variables by

$$
z=x+\mathrm{i} y
$$

Inspecting (221), we can put the Gaussian form

$$
f\left(x_{1}, y_{1}\right)=A \mathrm{e}^{-K x_{1}^{2}} \mathrm{e}^{-K y_{1}^{2}},
$$

without loss of generality. Then, the integral equation (221) reduces to

$$
\frac{1}{1+n(0)} \mathrm{e}^{-x^{2} /(1+n(0))} \mathrm{e}^{-y^{2} /(1+n(0))}=\frac{A}{K+1} \mathrm{e}^{-|\alpha|^{2} K /(K+1)},
$$


which determines the coefficients $A$ and $K$ as

$$
A=1 / n(0), \quad K=1 / n(0) .
$$

We finally have the c-number function corresponding to the initial state (105) with (106) in the form

$$
f(z, t=0)=f(z, z)=\frac{1}{n(0)} \mathrm{e}^{-|z|^{2} / n(0)} .
$$

The coefficients $a_{\ell}$ 's are obtained with the help of the initial condition in the form

$$
a_{\ell}=\frac{1}{n(0)} \int_{0}^{\infty} \mathrm{d} \zeta^{\prime} L_{\ell}\left(\zeta^{\prime}\right) \mathrm{e}^{-\zeta^{\prime} \bar{n} / n(0)}
$$

Substituting (227) into (218), we can derive the solution of (205) as

$$
\begin{aligned}
F(\zeta \bar{n}, t) & =\frac{1}{n(0)} \sum_{\ell=0}^{\infty} \int_{0}^{\infty} \mathrm{d} \zeta^{\prime} L_{\ell}\left(\zeta^{\prime}\right) R_{\ell}(\zeta) \mathrm{e}^{-\zeta^{\prime} \bar{n} / n(0)} \mathrm{e}^{-2 \kappa \ell t} \\
& =\frac{1}{n(0)} \sum_{\ell=0}^{\infty} \int_{0}^{\infty} \mathrm{d} \zeta^{\prime} L_{\ell}\left(\zeta^{\prime}\right) R_{\ell}(\zeta) \mathrm{e}^{-\zeta^{\prime}} \mathrm{e}^{-\zeta^{\prime}(\bar{n}-n(0)) / n(0)} \mathrm{e}^{-2 \kappa \ell t} \\
& =\frac{1}{\bar{n}} \sum_{\ell=0}^{\infty} \sum_{k=0}^{\infty} R_{\ell}(\zeta)\left(\frac{\bar{n}-n(0)}{\bar{n}}\right)^{k} \mathrm{e}^{-2 \kappa \ell t} \int_{0}^{\infty} \mathrm{d} \zeta^{\prime} L_{\ell}\left(\zeta^{\prime}\right) R_{k}\left(\zeta^{\prime}\right) \\
& =\frac{1}{\bar{n}} \sum_{\ell=0}^{\infty} R_{\ell}(\zeta)\left(\frac{\bar{n}-n(0)}{\bar{n}}\right)^{\ell} \mathrm{e}^{-2 \kappa \ell t} \\
& =\frac{1}{\bar{n}} \mathrm{e}^{-\zeta} \sum_{\ell=0}^{\infty} L_{\ell}(\zeta)\left(\frac{\bar{n}-n(0)}{\bar{n}} \mathrm{e}^{-2 \kappa t}\right)^{\ell} \\
& =\frac{1}{n(t)} \mathrm{e}^{-\xi / n(t)},
\end{aligned}
$$

with

$$
n(t)=\bar{n}+(n(0)-\bar{n}) \mathrm{e}^{-2 \kappa t} .
$$

For the second equality, $\mathrm{e}^{-\zeta^{\prime} \bar{n} / n(0)}$ was divided into two exponentials. For the third equality, we used the generating function (210) of the Laguerre polynomials for $\mathrm{e}^{-\zeta^{\prime}(\bar{n}-n(0)) / n(0)}$ and (215). For the fourth equality, the orthogonality (216) was used. For the final equality, we used the formulae (215) and (210), again. Note that $n(t)$ defined by (229) satisfies the Boltzmann equation (21) with the initial condition $n(t=0)=n(0)$.

Now, we can evaluate $(\alpha, \tilde{\beta}|0(t)\rangle$ as

$$
\begin{aligned}
(\alpha, \tilde{\beta}|0(t)\rangle & =\int \frac{\mathrm{d}^{2} z}{\pi} f(z, t)(\alpha, \tilde{\beta} \mid z, \tilde{z}) \\
& =\int \frac{\mathrm{d}^{2} z}{\pi} F(z, t)(\alpha, \tilde{\beta} \mid z, \tilde{z})
\end{aligned}
$$




$$
\begin{aligned}
& =\frac{1}{n(t)} \int \frac{\mathrm{d}^{2} z}{\pi} \mathrm{e}^{-|z|^{2} / n(t)}(\alpha, \tilde{\beta} \mid z, \tilde{z}) \\
& =\frac{1}{n(t)} \mathrm{e}^{-\frac{1}{2}|\alpha|^{2}-\frac{1}{2}|\beta|^{2}} \int \frac{\mathrm{d}^{2} z}{\pi} \mathrm{e}^{-|z|^{2}[n(t)+1] / n(t)+\alpha^{*} z+\beta z^{*}},
\end{aligned}
$$

which reduces to (104) and to (201) after the integration with respect to $z$.

\section{References}

1. Arimitsu T., Umezawa H. A general formulation of non-equilibrium thermo field dynamics. // Prog. Theor. Phys., 1985, vol. 74, p. 429-432.

2. Arimitsu T., Umezawa H. Non-equilibrium thermo field dynamics. // Prog. Theor. Phys., 1987, vol. 77, p. 32-52.

3. Arimitsu T., Umezawa H. General structure of non-equilibrium thermo field dynamics. // Prog. Theor. Phys., 1987, vol. 77, p. 53-67.

4. Arimitsu T. Quantum field theoretical method in non-equilibrium systems - nonequilibrium thermo field dynamics. - In: Lecture Note of the Summer School for Younger Physicists in Condensed Matter Physics, published in "Bussei Kenkyu", Kyoto, 1993, vol. 60 , p. 491-526.

5. Arimitsu T. A canonical formalism of dissipative quantum systems. Non-equilibrium thermofield dynamics. // Condensed Matter Physics, 1994, No. 4, p. 26-88 (http://www.icmp.lviv.ua/icmp/journal/zbirnyk.4/).

6. Kubo R. Thermal ionization of trapped electron. // Phys. Rev., 1952, vol. 86, p. 929937.

7. Kubo R., Toyozawa T. Application of the method of generating function to radiative transitions of trapped electron in a crystal. // Prog. Theor. Phys., 1955, vol. 13, p. $160-182$.

8. Arimitsu T., Willeboordse F.H., Iwasaki T. An analytical treatment of a localized electron-phonon system within non-equilibrium thermo field dynamics. Intensity distribution. // Physica A, 1992, vol. 182, p. 214-227.

9. Arimitsu T., Arimitsu N. Time-resolved optical spectrum for transient resonant light scattering. // Phys. Rev. E, 1994, vol. 50, p. 121-137.

10. Arimitsu T., Ban M. A note on the photon echo study of the solvable model of microscopic phase modulation. // J. Phys. Soc. Japan, 1984, vol. 53, p 74-75.

11. Arimitsu T., Ban M. A note on the second order optical process for a solvable model of microscopic phase modulation to intermediate state. // J. Phys. Soc. Japan, 1984, vol. 53 , p. $76-78$.

12. Ban M., Arimitsu T. A note on the analytical treatment of a localized electron-phonon system including the change of frequency. // J. Phys. Soc. Japan, 1984, vol. 53, p. 939 942.

13. Takahashi Y., Umezawa H. Thermo field dynamics. // Collect. Phenom., 1975, vol. 2, p. 55 .

14. Tominaga T., Ban M., Arimitsu T., Pradko J., Umezawa H. Spin relaxation in terms of thermo field dynamics. // Physica, 1988, vol. A149, p. 26-48.

15. Ban M., Arimitsu T. Thermo field dynamical approach to optical dephasing. // Physica, 1987, vol. A146, p. 89-125. 
16. Tominaga T., Arimitsu T., Pradko J., Umezawa H. A derivation of the laser master equation in terms of thermo field dynamics. // Physica, 1988, vol. A150, p. 97-117.

17. Iwasaki T., Arimitsu T., Willeboordse F.H. Transient resonant light scattering for a localized electron-phonon system within non-equilibrium thermo field dynamics. - In: Thermal Field Theories, eds. Ezawa E, Arimitsu T., Hashimoto Y., North-Holland, 1991, p. 459-468.

18. Arimitsu T., Sudo Y., Umezawa H. Dynamical rearrangement of the thermal vacuum in thermo field dynamics. // Physica, 1987, vol. A146, p. 433-451.

19. Arimitsu T., Guida M., Umezawa H. Spontaneous creation of dissipation in thermo field dynamics. // Europhys. Lett., 1987, vol. 3, p. 277-282.

20. Arimitsu T., Guida M., Umezawa H. Dissipative quantum field theory. Field dynamics. // Physica, 1988, vol. A148, p. 1-26.

21. Arimitsu T., Umezawa H. Dissipative quantum field theory - spontaneous creation of dissipation in TFD. - In: Advances on Phase Transitions and Disordered Phenomena, eds. Busiello G., De Cesare L., Mancini F., Marinaro M., World Scientific, Singapore, 1987, p. 483-504.

22. Umezawa H., Arimitsu T. Spontaneous creation of dissipation in thermo field dynamics. - In: Foundation of Quantum Mechanics - In the Light of New Technology, eds. Namiki M., Ohnuki Y., Murayama Y., Nomura S. Physical Society of Japan, Tokyo, 1987, p. 79-90.

23. Arimitsu T., Umezawa H., Yamanaka Y., Papastamatiou P. Spontaneous creation of dissipation in thermo field dynamics and its examples. // Physica, 1988, vol. A148, p. $27-45$.

24. Arimitsu T. Migration of unstable vacuum for dissipative systems. // RIMS Report, Kyoto Univ., 1998, vol. 1099, p. 70-95.

25. Arimitsu T., Endo Y. Non-demolition continuous measurement and the quantum stochastic differential equations. // RIMS Report, Kyoto Univ., 1998, vol. 1066, p. 2536 .

26. Belavkin V.P., Staszewski P. Nondemolition observation of a free quantum particle. // Phys. Rev. A, 1992, vol. 45, p. 1347-1356.

27. Tokarchuk M.V, Arimitsu T., Kobryn A.E. Thermo field hydrodynamic and kinetic equations of dense quantum nuclear systems. // Condensed Matter Physics, 1998, vol. 1 , p. 605-642. .

28. Arimitsu T. Thermal processes in the hydrodynamics stage in terms of non-equilibrium thermo field dynamics. // J. Phys. A: Math. Gen., 1991, vol. 24, p. L1415-L1421.

29. Zubarev D.N., Tokarchuk M.V. Nonequilibrium thermo field dynamics and nonequilibrium statistical operator method. // Teor. Mat. Fiz., 1991, vol. 87, p. 286-310 (in Russian).

30. Sudarshan E.C.G. Equivalence of semiclassical and quantum mechanical descriptions of statistical light beams. // Phys. Rev. Lett., 1963, vol. 10, p. 277-279.

31. Glauber R.J. Coherent and incoherent states of the radiation field. // Phys. Rev., 1962, vol. 131, p. 2766-2788.

32. Agarwal G.S, Wolf E. Calculus for functions of noncommuting operators and general phase-space methods in quantum mechanics. I. Mapping theorems and ordering of functions of noncommuting operators. // Phys. Rev. D, 1970, vol. 2, p. 2161-2186; Calculus for functions of noncommuting operators and general phase-space methods in quantum mechanics. II. Quantum mechanics in phase space. // Phys. Rev. D, 
1970, vol. 2, p. 2187-2205; Calculus for functions of noncommuting operators and general phase-space methods in quantum mechanics. III. A general Wick theorem and multitime mapping. // Phys. Rev. D, 1970, vol. 2, p. 2206-2225.

33. Haake F. Statistical treatment of open systems by generalized master equations. - In: Springer Tracts in Modern Physics, vol. 66, Springer-Verlag, 1973, p. 98-168.

\title{
Застосування нерівноважної термопольової динаміки для опису систем з локалізованим електроном і фононами
}

\author{
Н.Аріміцу ${ }^{1}$, Т.Аріміцу ${ }^{2}$ \\ 1 Відділ комп'ютерної інженерії \\ Національного університету Йокогама, \\ Токіваваі, Йокогама 240-8501, Японія \\ 2 Інститут фізики Університету Цукуба, Ібаракі 305-8571, Японія
}

Отримано 6 жовтня 1999 р.

За допомогою операторної алгебри в рамках нерівноважної термопольової динаміки (НТД) досліджується адсорбційний спектр аналітично розв'язуваної моделі локалізованого електрона і фононів. Знаходження й поведінка адіабатичних потенціалів для основного та збуджених електронних станів вважаються відмінними один від одного. Структури адсорбційних спектрів досліджуються для різних випадків, у тому числі для повільних та швидких модуляцій. Пояснюється самодостатність методу НТД для розв'язку задачі.

Ключові слова: нерівноважна термопольова динаміка, локалізований електрон, адіабатичний потенціал, адсорбційний спектр, швидка й повільна модуляції

PACS: 05.30. $-d, 42.50 . c t$ 